\title{
The Role of Wound Swab Microbial Cultures and Antibiotic Sensitivity Results in the Management of Sacrococcygeal Pilonidal Abscess
}

\author{
Chao Cheng *, Jiun Miin Lai, Krinalkumar Mori \\ Department of Surgery, Northern Health, VIC, Australia
}

\section{Article history:}

Received: June 27, 2019

Revised: September 24, 2019

Accepted: September 24, 2019

*Corresponding Author:

Chao Cheng

Department of Surgery, Northern Health,

VIC, Australia

E-mail: candy.chaocheng@gmail.com

ORCID

Chao Cheng

https://orcid.org/0000-0003-3180-7854

Krinalkumar Mori

https://orcid.org/0000-0001-8513-2130

\section{Introduction}

Pilonidal sinus disease refers to a condition that affects approximately $0.7 \%$ of the population [1] where there is a small hole or tunnel in the skin often around the hair follicle in the natal cleft, forming a midline pit [2]. It can present acutely (or acutely recurrent within 6 weeks) where treatment involves draining the pus from the abscess or present as a chronic infection where the skin and tissues containing the sinus is removed. The condition, and treatment for pilonidal sinus often burden patients and caretakers with wound care and frequent visits to general practitioners (GPs) or hospital outpatient clinics [3]. For patients who present to hospitals with acute pilonidal abscess, the current practice is surgical drainage combined with antibiotic therapy. Wound swabs are routinely taken in the peri-operative period, and antibiotics are commonly initiated empirically pending culture and sensitivity results [2]. There are only a few studies that have reported observations on pilonidal abscess in the sacrococcygeal area, however, those studies that addressed superficial skin abscesses reported a limited role of wound culture results [4,5]. One study concluded that the use of postoperative antibiotics was guided by the patient's clinical picture, and that the microorganisms cultured were predictable, and sensitive to empirical antibiotics used in their institution [4].

The primary objective of this study was to determine the role of wound swabs in the management antibiotic therapy in patients with pilonidal abscess by analyzing the proportion of 
patients with acute pilonidal abscess whose initial prescription of antibiotics was changed based on the wound swab results. The secondary objective of this study was to identify the common microorganisms cultured from patients with acute pilonidal abscess.

\section{Materials and Methods}

\section{Study design}

The study was a single center retrospective analysis conducted at The Northern Hospital (TNH), Victoria. It included patients who presented to TNH Emergency Department (ED) with acute sacrococcygeal pilonidal abscess between 1st January 2013 to 30th June 2016 and were admitted under the Acute General Surgical Unit. These patients subsequently underwent incision and drainage of the abscess in the operating theatre.

With the help from the medical records department at $\mathrm{TNH}$, patients were identified using diagnostic and procedural coding such as "pilonidal cyst with abscess," "pilonidal cyst without abscess," "incision of pilonidal sinus or cyst," "excision of pilonidal sinus or cyst," "aspiration of abscess of skin and subcutaneous tissue," and "incision and drainage of abscess of skin and subcutaneous tissue."

Patient information was then collected retrospectively using the hospital electronic medical record. The following information was collected: emergency case or elective case, first episode or re-presentation, intravenous antibiotics started upon admission, wound swabs and the results of the wound swab, oral antibiotics on discharge, outpatient clinic follow-up, and details of antibiotic change if any.

The study protocol ALR 16.2016 was reviewed and approved by the Low and Negligible Risk Ethics Committee at the Northern Hospital.

\section{Inclusion criteria}

Patients were included in the study who presented to ED with acute sacrococcygeal pilonidal abscess and were admitted under Acute General Surgical Unit for incision and drainage of the abscess in theatre from $1^{\text {st }}$ January 2013 to $30^{\text {th }}$ June 2016. Re-presentations or recurrence of pilonidal abscesses during the study period were included as separate admissions.

\section{Exclusion criteria}

Patients who were admitted to hospital for treatment of acute abscesses not in the sacrococcygeal region were excluded from this study. Patients who were admitted under a general surgical inpatient unit for an elective procedure, such as excision of pilonidal sinus, lay open of pilonidal sinus, Karydakis procedure, or modified Karydakis procedure were also excluded from this study.

\section{Outcome measures}

The primary outcome of the study was any change in antibiotics prescribed to a patient with acute sacrococcygeal pilonidal abscess based on the results of the wound swab that was taken peri-operatively. The secondary outcome was a positive wound swab where microorganisms were identified and sensitivities to the antibiotics were reported by the laboratory.

\section{Results}

During the study period from January 2013 to June 2016, there were 464 cases identified. Among those 464 cases, duplicated admissions, elective cases, and abscesses other than pilonidal abscesses were excluded from the study. There were 297 cases included in this study that met the inclusion criteria (Figure 1).

\section{Patient demographics}

Of the 297 cases, 85 were female $(85 / 297,28.6 \%)$ and 212 were male $(212 / 297,71.4 \%)$. The average age of the patients was 27 years (ranging from $15-70$ years).

\section{Antibiotic therapy}

Among the 297 cases included in the study, 283 (283/297, 95.3\%) were prescribed intravenous antibiotics on admission prior to surgical intervention. Commonly used antibiotic therapy including a combination of cephazolin and metronidazole (95/283,33.6\%), and cephazolin alone (85/283, $30 \%)$. There were 224 cases out of 297 (224/297, 75.4\%) that had a wound swab taken in theatre during the operation. 151 cases $(151 / 297,50.8 \%)$ were post-operatively prescribed oral

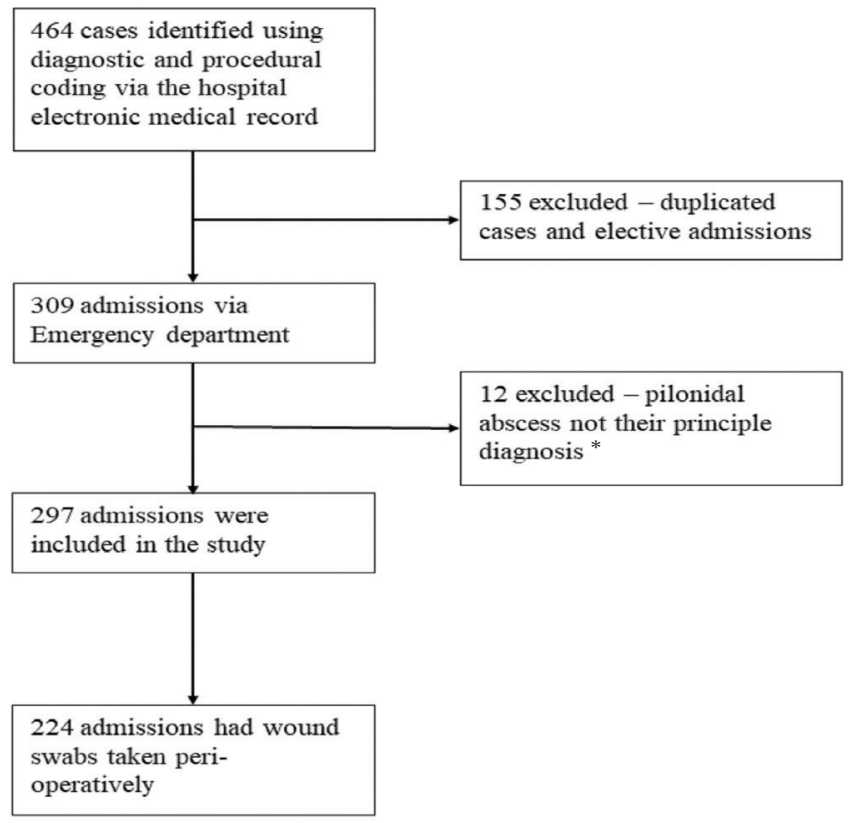

Figure 1. flow diagram of the study population.

* Details of other exclusions: chronic pilonidal sinus excision (4 cases); gluteal/buttock abscesses ( 3 cases); post-operative wound infection re-admission (1 case); haemorrhoidectomy (1 cases); axillary sinus excision ( 1 case); and perianal abscess ( 2 cases). 
antibiotics upon discharge, and the most prescribed antibiotic was Augmentin Duo Forte (89/151, 60.0\%).

Amongst the 224 cases that had wound swabs taken from the incised abscess, 130 cases $(130 / 224,58 \%)$ were followed up in the general surgical clinic at TNH, 35 cases $(35 / 224$, $15.6 \%)$ were discharged to their local GPs, and 59 cases (59/224, 26.3\%) failed to attend the follow-up appointments. The follow-up period ranged from 1 week to 26 weeks, and majority of patients were followed up 2-4 weeks, and median follow-up period was 5 weeks. There were 15 patients who were reviewed within 1 week either due to the size of the wound being large or the patient was discharged where a vacuum dressing was used. All 15 patients (15/130, 11.5\%) were still on oral antibiotics at the time of post-operative review. Out of the 130 cases who had wound swabs taken and were followed up in surgical clinic, there were 7 wound swab results documented in the outpatient notes. One patient $(1 / 130,0.8 \%)$ was prescribed a different antibiotic in clinic based on the results of the culture of the swab and sensitivity to antibiotics. This patient was given intravenous flucloxacillin peri-operatively and discharged home with oral flucloxacillin. In the follow-up appointment in clinic, it was documented that the wound was healing well and the wound swabs cultured grew mixed anaerobic microorganisms. The patient was then prescribed a course of oral metronidazole upon discharge from clinic for further follow-up by the patients GP. This patient did not present again to this hospital for recurrent acute pilonidal abscess during the study period.

\section{Common microorganism}

Results from swab culture and sensitivity to antibiotics for 224 cases were collated (Table 1). Among the 224 wound swabs, 194 (194/224, 86.6\%) had microorganisms that grew on culture and $30(30 / 224,13.4 \%)$ had no growth after 48 hours of incubation. There were 33 cases $(33 / 224,14.7 \%)$ that had microorganisms identified and sensitivities to antibiotics reported by the laboratory. The most common types of microorganisms identified were mixed anaerobic

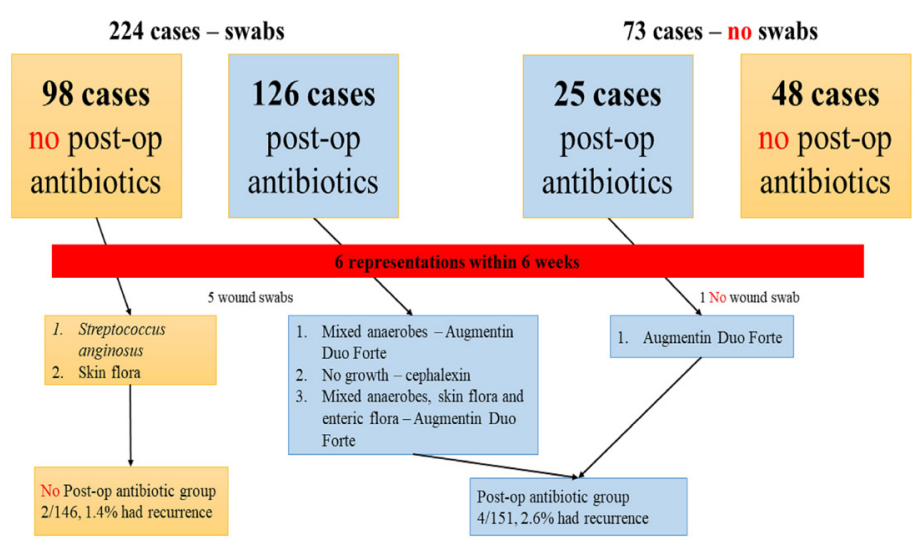

Figure 2. Flow chart and details of representation cases. microorganisms (138/224, 61.6\%) and skin flora (45/224, 20.1\%). Antibiotic sensitivities were not provided for these common types, as they are usually covered by empirical antibiotics.

\section{Re-presentation}

There were 6 patients $(6 / 297,2 \%)$ who had recurrent presentation with another episode of acute sacrococcygeal pilonidal abscess within a 6-week period, 5 of which had wound swabs taken in theatre during their first presentation (Figure 2). Three patients out of the 6 re-presentations had wound swabs taken and were discharged with oral antibiotics for their initial presentation. Two of these 3 wound swabs when cultured identified mixed anaerobic microorganisms, and both patients were given oral Augmentin Duo Forte postoperatively. The remaining wound swab had no growth in culture, and the patient was prescribed oral cephalexin postoperatively.

Two patients out of the 6 re-presentations had wound swabs taken intra-operatively, however were not prescribed antibiotics on discharge during their first presentation. One wound swab culture grew skin flora and the other grew Streptococcus anginosus. None of the 5 wound swabs of the re-presentation cases (5/6) when cultured, yielded antibiotic sensitivity. All 6 recurrent presentations were taken back to the operating theatre for another incision and drainage regardless of whether a wound swab was taken or whether the swab had yielded growth of microorganisms during the prior admission. Wound swab culture from the first presentation did not change the antibiotic choice nor the surgical management when they re-presented.

\section{Discussion}

Patients who present to ED with acute sacrococcygeal pilonidal abscess, routinely have wound swabs taken perioperatively. This study showed that most patients (75.4\%) who presented to ED with acute sacrococcygeal pilonidal abscess had wound swabs taken. One retrospective study on all cutaneous abscesses reported that $67.5 \%$ of their patients had wound specimens sent to the laboratory from the procedure [5]. Another study reported that $100 \%$ of their patients had wound swabs taken at the procedure which was assumed from a microbiology database [4]. In this current study, of the $75.4 \%$ of patients that had wound swabs taken, $87.1 \%$ had microorganism growth in culture and $12.9 \%$ had no growth after 48 hours of incubation. This result is comparable to Khan's study where $70.9 \%$ cases had microorganism growth in culture [4], however Khan's study included all superficial soft tissue abscesses whereas this study focused on sacrococcygeal pilonidal abscesses.

Culture of microorganisms from a wound and testing their sensitivities to antibiotics is the standard practice for treating infections, and it is used to help with antibiotic selection 
Table 1. Details of wound swabs.

\begin{tabular}{|c|c|c|c|c|c|}
\hline $\begin{array}{l}\text { Microorganisms identified } \\
\text { on culture }\end{array}$ & $\begin{array}{l}\text { Patient } \\
\text { Numbers } \\
(N=224 \%)\end{array}$ & $\begin{array}{c}\text { Patient } \\
\text { demographics }\end{array}$ & $\begin{array}{l}\text { Most commonly used } \\
\text { Pre-operative antibiotics }\end{array}$ & $\begin{array}{l}\text { Most commonly used } \\
\text { Post-operative antibiotics }\end{array}$ & $\underset{*}{\text { Recurrence }}$ \\
\hline Mixed anaerobes & $138(61.6)$ & $\begin{array}{l}\text { Male } 88 \\
\text { Female } 50\end{array}$ & Cephazolin + metronidazole $(43 / 138)$ & Augmentin duo forte $(41 / 138)$ & 2 \\
\hline Skin flora & $45(20.1)$ & $\begin{array}{c}\text { Male } 34 \\
\text { Female } 11\end{array}$ & Cephazolin + metronidazole $(17 / 45)$ & Augmentin duo forte (17/45) & 2 \\
\hline $\begin{array}{l}\text { No growth after } 24 \text { hours of } \\
\text { incubation }\end{array}$ & $30(13.4)$ & $\begin{array}{l}\text { Male } 20 \\
\text { Female } 10\end{array}$ & Cephazolin + metronidazole $(12 / 30)$ & Augmentin duo forte $(9 / 30)$ & 1 \\
\hline $\begin{array}{l}\text { Streptococcus milleri/ } \\
\text { anginosus group }\end{array}$ & $10(4.5)$ & Male 10 & $\begin{array}{l}\text { Cephazolin }(3 / 10) \\
\text { Cephazolin }+ \text { metronidazole }(3 / 10)\end{array}$ & Augmentin duo forte (3) & \\
\hline $\begin{array}{l}\text { Staphylococcus aureus } \\
\text { (MSSA) }\end{array}$ & $9(4.0)$ & $\begin{array}{l}\text { Male } 7 \\
\text { Female } 2\end{array}$ & Cephazolin (4/9) & Augmentin duo forte (3/9) & \\
\hline Enteric flora & $7(3.1)$ & $\begin{array}{l}\text { Male } 4 \\
\text { Female } 3\end{array}$ & Cephazolin + metronidazole $(7 / 7)$ & Augmentin duo forte (3/7) & 1 \\
\hline $\begin{array}{l}\text { Staphylococcus aureus } \\
\text { (MRSA) }\end{array}$ & $2(0.9)$ & Male 2 & $\begin{array}{l}\text { Cephazolin + metronidazole }(1) \\
\text { Flucloxacillin + metronidazole }(1)\end{array}$ & Augmentin duo forte $(2 / 2)$ & \\
\hline Streptococcus Group G & $2(0.9)$ & Male 2 & $\begin{array}{l}\text { Flucloxacillin (1) } \\
\text { Cephazolin + metronidazole (1) }\end{array}$ & $\begin{array}{l}\text { Flucloxacillin (1) } \\
\text { Augmentin duo forte (1) }\end{array}$ & \\
\hline Streptococcus Group C & $2(0.9)$ & Male 2 & Cephazolin + metronidazole & $\begin{array}{l}\text { Augmentin duo forte (1) } \\
\text { Cephalexin (1) }\end{array}$ & \\
\hline Bacteroides fragilis & $2(0.9)$ & Male 2 & $\begin{array}{l}\text { Ceftriaxone (1) } \\
\text { Cephazolin }+ \text { metronidazole ( } 1)\end{array}$ & $\mathrm{N} / \mathrm{A}$ & \\
\hline Streptococcus pyogenes & $1(0.4)$ & Male & Cephazolin + metronidazole & Augmentin duo forte & \\
\hline Clostridium ramosum & $1(0.4)$ & Female & Ceftriaxone + metronidazole & & \\
\hline $\begin{array}{l}\text { Coagulase negative } \\
\text { Staphylococcus }\end{array}$ & $1(0.4)$ & Male & Flucloxacillin & Flucloxacillin & \\
\hline Proteus species & $1(0.4)$ & Male & $\mathrm{N} / \mathrm{A}$ & $\mathrm{N} / \mathrm{A}$ & \\
\hline Streptococcus intermedius & $1(0.4)$ & Male & Cephazolin + metronidazole & $\mathrm{N} / \mathrm{A}$ & \\
\hline $\begin{array}{l}\text { Staphylococcus } \\
\text { lugdunensis, }\end{array}$ & $1(0.4)$ & Male & Cephazolin + metronidazole & Cephalexin & \\
\hline Klebseilla pneumoniae & $1(0.4)$ & Female & Cephazolin + metronidazole & Augmentin duo forte & \\
\hline $\begin{array}{l}\text { Peptostreptococcus } \\
\text { anaerobius }\end{array}$ & $1(0.4)$ & Male & Cephazolin & Augmentin duo forte & \\
\hline E. coli & $1(0.4)$ & Male & Cephazolin & Augmentin duo forte & \\
\hline Diphtheroids & $1(0.4)$ & Female & Cephazolin + metronidazole & & \\
\hline Streptococcus anginosus & $1(0.4)$ & Male & Cephazolin & & 1 \\
\hline
\end{tabular}

* The swab taken from one patient may culture multiple different microorganisms.

[4]. The most common microorganisms identified in culture in this current study was mixed anaerobic microorganisms (61.6\%), a finding similar to other studies [3,4]. Although a large proportion of the wound swabs taken grew microorganisms in culture, only $14.7 \%$ were given specific antibiotic sensitivities. Among the small group of patients who re-presented to ED within 6 weeks from a previous admission for pilonidal abscesses, wound swab results did not demonstrate resistant organisms. Mixed anaerobic microorganisms and skin flora were still the most common.

Traditionally, empirical intravenous antibiotic therapy is prescribed due to skin cellulitis, increased inflammatory markers, or patients who have sepsis on presentation. In line with wound swab results in this current study and previous studies, current guidelines recommend using a secondgeneration cephalosporin and metronidazole (to treat anaerobic microorganisms) when prescribing antibiotics to patients with pilonidal abscess peri-operatively [6]. Australian therapeutic guidelines do not have a separate section for sacrococcygeal pilonidal abscesses, as they are covered under the management for boils and carbuncles. The key therapeutic intervention recommended is incision and drainage, and 
antibiotic therapy using dicloxacillin or flucloxacillin [7]. The data in this study suggests that the majority of patients (63.3\%) were prescribed second generation cephalosporin, and $33.3 \%$ were prescribed metronidazole to treat anaerobic microorganisms.

Post-operatively, patients were commonly discharged prior to the wound swab results becoming available [2,4]. Half of the study population (50.8\%) were given oral antibiotics on discharge. There was no identifiable correlation that a wound swab taken affected post-operative antibiotic use. The majority of patients who were prescribed antibiotics at discharge were covered in the treatment for anaerobic microorganisms. Due to the retrospective nature of the study, the post-operative antibiotic therapy prescribed at discharge could not be identified. In this current study, the cohort of patients who did not receive antibiotics on discharge did not have a high recurrent rate compared to those who were prescribed oral antibiotics on discharge. However, data was not collected to determine whether those patients who were given antibiotics were more medically complex such as patients who are immunosuppressed, or whether there was significant cellulitis surrounding the wound which could potentially delay healing.

Of the patients who had wound swabs taken (75.4\%), and were followed up in the outpatient clinic at this hospital, there were $0.8 \%$ of patients given a different antibiotic therapy based on the laboratory microorganism culture results. As a retrospective study, the outcome of patients who were followed up in the community could not be analyzed, nor could it be determined whether the results of wound swabs were followed up for all patients in the outpatient clinic, based on the electronic outpatient progress notes. Furthermore, the patient who was prescribed a different antibiotic in clinic had no further follow-up or re-presentation to this hospital within 6 weeks of the first acute presentation. It could not be determined whether the change in antibiotic therapy played a significant role in wound healing or prevention of recurrence. Evidently, the focus of post-operative review in the outpatient setting were symptoms and the progress of the wound healing, which guided further clinical follow-up and management. Importantly, this study showed that the management of representation of a patient with a pilonidal abscess within 6 weeks of treatment of the initial abscess, was treatment with further surgical drainage. In addition, the laboratory results of wound swabs cultured from the previous abscess did not play a role in the management of the subsequent abscess.

The laboratory costs for the culture of the wound swab and antibiotic sensitivity costs $\$ 6.4$ (Australian dollars) for processing and microscopy, and $\$ 33.75$ for microorganism culture and antibiotic sensitivity analysis. In this study there were 297 patients assessed over 3 and half years, and the recent assessment estimated 140 presentations with acute sacrococcygeal pilonidal abscesses within 1 calendar year. By not requesting wound swabs and antibiotic sensitivity assays on patients with pilonidal abscesses, the hospital may potentially save $\$ 5,000$ per calendar year.

If the laboratory tests to culture wound swabs from pilonidal abscesses and perform antibiotic sensitivity were not requested this may save $\$ 5,000$ per calendar year.

\section{Limitations}

Limitations of the study lie mainly with the study design. It is a retrospective study limiting the assessment of antibiotic therapy prior to presentation at hospital. Prior use of antibiotics prescribed by GPs may affect wound swab laboratory tests. Additionally, patients included in the study were not all followed up in the surgical outpatient clinic of this hospital. Family doctors may have followed up the results from the wound swab and prescribed further antibiotic therapy. Information collection for this study relied entirely on documentation and quality of notes taken during the audit at The Northern Hospital (TNH), Victoria which were highly variable.

\section{Conclusion}

Wound swabs could be omitted during peri-operative care for patients with acute sacrococcygeal pilonidal abscess as the results from wound swabs did not change the management of these patients. Omitting wound swabs may save the health care system around $\$ 5,000$ per calendar year. Preoperative antibiotic therapy should include an antibiotic to treat anaerobic microorganisms because this was commonly cultured from wound swabs from acute sacrococcygeal pilonidal abscesses.

\section{Conflict of Interest}

No potential conflict of interest relevant to this article was reported.

\section{References}

[1] Shabbir J, Chaudhary BN, Britton DC. Management of sacrococcygeal pilonidal sinus disease: a snapshot of current practice. Int J Colorectal Dis 2011;26(12):1619-20.

[2] de Parades V, Bouchard D, Janier M, Berger A. Pilonidal sinus disease. J Visc Surg 2013;150(4):237-47.

[3] Humphries AE, Duncan JE. Evaluation and management of pilonidal disease. Surg Clin North Am 2010;90(1):113-24.

[4] Khan MN, Vidya R, Lee RE. The limited role of microbiological culture and sensitivity in the management of superficial soft tissue abscesses. ScientificWorldJournal 2006;6:1118-23.

[5] Payne CJ, Walker TW, Karcher AM, Kingsmore DB, Byrne DS. Are routine microbiological investigations indicated in the management of nonperianal cutaneous abscesses? Surgeon 2008;6(4):204-6.

[6] Johnson EK [Internet]. Pilonidal disease. Waltahm (MA):UpToDate Inc. [cited 2019 Sep 20]. Available from: https://www.uptodate.com/ contents/pilonidal-disease?search=pilonidal\%20abscess\&source=search result\&selectedTitle=1 22\&usage_type=default\&display_rank=1.

[7] eTG complete [Internet]. Boils and Carbuncles. Melbourne (Australia): Therapeutic Guideline Ltd; 2019 [updated 2019 Apr; cited 2019 Sep 20]. Available from: https://tgldcdp.tg.org.au.acs.hcn.com.au/ viewTopic?topicfile=boils-carbuncles\#toc_d1e47. 